\title{
Identification of Aurora Kinase A as a Biomarker for Prognosis in Obesity Patients with Early Breast Cancer
}

This article was published in the following Dove Press journal: OncoTargets and Therapy

\author{
Junhan Jiang (1D \\ Zihe Guo' \\ Junnan $\mathrm{Xu} \mathbb{D}^{2}$ \\ Tao Sun $\left(\mathbb{D}^{2}\right.$ \\ Xinyu Zheng $\mathbb{D}^{1,3}$
}

'Department of Breast Surgery, The First Affiliated Hospital of China Medical University, Shenyang, People's Republic of China; ${ }^{2}$ Department of Breast Medical,

Cancer Hospital of China Medical University, Liaoning Cancer Hospital, Shenyang, People's Republic of China; ${ }^{3}$ Laboratory I, Cancer Institute, The First Affiliated Hospital of China Medical University, Shenyang, People's Republic of China
Correspondence: Xinyu Zheng Department of Breast Surgery, The First Affiliated Hospital of China Medical University, No. 155 Nanjing North Street, Heping District, Shenyang, Liaoning I I000I, People's Republic of China Tel/Fax +86248328 274l

Email xyzheng@cmu.edu.cn
Background: Obesity is associated both with a higher risk of developing breast cancer, particularly in postmenopausal women, and with worse disease outcome for women of all ages. Previous investigation suggested Aurora A kinase was able to partially restore the functionalities of obese adipose-derived mesenchymal stem cells by stabilizing their primary cilia and reestablishing a balance of multiple stemness-associated genes. The association between Aurora A and obesity breast cancer is still unclear. We hypothesized that overexpression of Aurora A was associated with poor survival in obesity breast cancer and the related axis mechanism was involved.

Methods: A total of 517 primary breast cancer specimens were collected from the First Affiliated Hospital of China Medical University between January 2011 and November 2016. Our independent variable was BMI at baseline, categorized as overweight (BMI $\geq 25 \mathrm{~kg} / \mathrm{m}^{2}$, as obesity cohort), and normal (18.5 $\leq \mathrm{BMI}<25 \mathrm{~kg} / \mathrm{m}^{2}$, as non-obesity cohort). The immunohistochemical (IHC) staining was performed with Aurora A, Survivin, MMP11, Cyclin B1, and Cathepsin L. Kaplan-Meier curve was used to analyze overall survival in our cohorts and TCGA-BRCA data (GSE3494). Log rank test was used to calculate $P$ values. Protein-protein interaction (PPI) network analysis and MCODE model were used to analyze the Aurora-altered signal pathway from GSE78958.

Results: Among 517 breast patients, Aurora A-positive (staining scores $\geq 4$ ) was significantly higher in obesity breast carcinoma compared with non-obesity cancer carcinoma $\left(\chi^{2}=9.79\right.$, $P=0.002)$, with more frequency in hormone receptor-negative $(68.4 \%$ vs $77.9 \%, P=0.015)$ and HER2-positive patients $(28.7 \%$ vs $17.9 \%, P=0.003)$. High Aurora A expression was remarkably and significantly associated with overall survival (OS) (8-year OS ratio: 69.5\% vs 81.1\%, $\mathrm{OR}=1.76,95 \% \mathrm{CI}: 1.03 \sim 3.02, P=0.041$ ) in obesity cohort. Interestingly, higher expression of Aurora A was not associated with a shorter overall survival time among the non-obesity breast cancer ( 8 -year OS ratio: $81.4 \%$ vs $85.8 \%, \mathrm{OR}=1.40,95 \% \mathrm{CI}$ : $0.79 \sim 2.45, P=0.229$ ). As for RFS, the expression levels of Aurora A expression genes have no significance with RFS statistically in nonobesity and obesity patients. Aurora A and lymph node metastases were significantly poor prognostic factors for OS, and borderline significance was noted for high BMI. Kaplan-Meier survival analysis from TCGA database confirmed that the high Aurora A expression group had worse prognosis $(\mathrm{HR}=1.47,95 \% \mathrm{CI}: 1.14-1.90, P=0.003)$. The KEGG pathway enrichment results were consistent with GO biological process term analysis, in which CCNB1 was enriched for upregulated Aurora A. In our samples, Aurora A level on tumor cytoplasm had broad connections with Cyclin B1 by IHC correlation analysis (correlation coefficient $=0.227, P=0.001$ ).

Conclusion: Our finding demonstrates here for the first time that high expression of Aurora A was notably correlated with early recurrence and poor overall survival in obesity patients with early breast cancer. The Aurora A-Cyclin B1 axis could be a potential promising therapeutic target for cancer intervention and therapy.

Keywords: early breast cancer, obesity, Aurora A, overall survival, Cyclin B1 


\section{Background}

Breast cancer is the most common cancer and one of the leading causes of cancer death in female s world wide and accounts for $15 \%$ of all newly female diagnosed cancer in the People's Republic of China. ${ }^{1}$ Breast cancer is a greatly heterogeneous disease and categorized by distinct intrinsic subtypes based on immunohistochemical biomarkers, estrogen receptor (ER), progesterone receptor (PR), human epidermal growth factor receptor 2 (HER2) and Ki-67 index. ${ }^{2}$ Despite advances in target therapy, endocrine therapy and chemotherapy, thousands of patients have died of breast cancer-specific relapses or distant metastasis. ${ }^{2}$ We need to investigate novel biomarkers to improve prognosis and guide precision therapy, especially in special populations, including obesity patients.

Recent decades have suggested overweight/obesity is becoming increasingly prevalent globally. ${ }^{3}$ This trend is also seen in Asia including the People's Republic of China and females have progressively high prevalence. Based on World Health Organization (WHO) Criterion, overweight/ obesity is defined as a body mass index (BMI, calculated as weight $(\mathrm{kg}) /$ height $\left.\left(\mathrm{m}^{2}\right)\right)$ greater than $25 \mathrm{~kg} / \mathrm{m}^{2}(25.0-$ 29.9, overweight; 30.0-34.9, grade I; 35.0-39.9, grade II; and grade III equal to or greater than 40$).{ }^{4,5}$ Obesity is associated with increased risk of many fatal diseases including cardiovascular disease, stroke, diabetes and increased risk of cancer, especially breast cancer in postmenopausal patients. ${ }^{6}$ This link between obesity and cancer is built on many risk factors including elevated inflammation cytokine and adipokines secretion and is very complex. Abdominal adiposity plays an important role in generating a developing tumor environment. ${ }^{7}$

The link between obesity and breast cancer has been extensively investigated. ${ }^{4}$ Obesity is associated with an increased risk of ER-positive breast cancer in postmenopausal patients and greater mortality for both premenopausal (RR, 1.75; 95\% CI, 1.26-2.41) and postmenopausal (RR, 1.34; 95\% CI, 1.18-1.53) breast cancer. ${ }^{8}$ In postmenopausal women, the adipose tissue in obese people produces a higher level of tumor-promoting hormones, such as estrogen and leptin, which functions as an endocrine organ. However, in premenopausal women, the link between obesity and increased risk of breast cancer is still debatable. Two similar meta-analyses demonstrated that breast cancer risk was reduced by approximately $5 \%$ $\sim 8 \%$ per $5 \mathrm{~kg} / \mathrm{m}^{2}$ BMI increase in premenopausal women in 2.5 million and 0.3 million premenopausal population, respectively. ${ }^{9,10}$ However, the inverse study provided the point that a two-fold increase in breast cancer mortality is associated with a higher BMI index $\left(\right.$ BMI $>40 \mathrm{~kg} / \mathrm{m}^{2}$ vs BMI 18 24.9 kg/m²: RR=2.12; 95\% CI, 1.41 3.19). The higher levels of estrogen from the ovaries caused a reduction of gonadotrophin release by a negative feedback mechanism on the hypothalamic pituitary axis. ${ }^{11}$

The investigations demonstrated that obesity was also associated with aggressive tumor characteristics (bigger tumor size, more lymph node-positive) with more obvious trends in triple-negative breast cancer (TNBC). ${ }^{12}$ Previous studies focused on hormone receptor-positive breast cancer, and TNBC accounts for $10 \% 20 \%$ in all breast cancer types and presents a shorter survival than most other breast cancer subtypes. ${ }^{13}$ Although several small studies have demonstrated that increased triple-negative breast cancer risk and worse survival was indicated in obesity premenopausal women. ${ }^{14}$ Although the clinical outcomes for obesity patients with breast cancer are poor, the explanation suggests using the same therapy often with less efficacious results due to dose-limiting toxicities and obesity-related complications. Thus, we need biomarkers to identify the worse prognosis obesity patients and guide specific therapies for improving outcomes in obesity patients.

The molecular mechanisms that link obesity breast cancer are complex, an association between obesity and increased local and circulating pro-inflammatory/proangiogenic cytokines has been reported, ${ }^{4}$ which stimulate the most malignant cancer stem cell population to drive cancer growth, invasion, and metastasis. Aurora kinases (A, B and C) are key regulators of mitosis and multiple signaling pathways. ${ }^{15}$ Previous investigations suggested Aurora A kinase was able to partially restore the functionalities of obese adipose-derived mesenchymal stem cells. Gene amplification and protein overexpression of Aurora kinases A were consistently associated with cancer development in both hematologic malignancies and solid tumors and deregulation of Aurora kinases has been linked to tumorigenesis. ${ }^{15}$ Aurora A was indicated to function as a prognostic marker for worse outcome in ER-positive and lymph node-negative breast cancer ${ }^{16}$ and could surrogate Ki67 index, a proliferation marker widely used in clinical situations. However, how Aurora A outperforms proliferation markers in obesity patients with early breast cancer is still unclear. Screening analyses identified the dual Aurora $\mathrm{A}$ and B inhibitor JNJ-7706621 as a preferential inhibitor to overcome tamoxifen and aromatase inhibitor resistance. ${ }^{17}$ Regarding the growing knowledge on Aurora A mechanisms in breast cancer carcinogenesis, the ongoing development of 
targeting Aurora A signaling mechanisms will open new promising approaches for breast cancer prevention and treatment, specially in obesity patients.

Obesity is associated both with a higher risk of developing breast cancer, particularly in postmenopausal women, and with worse disease outcome for women of all ages. ${ }^{18}$ The association between Aurora A and obesity breast cancer is still unclear. In this work, we attempted to reveal the significance of Aurora A expression in obesity early breast cancer and the mechanisms related to obesity-related breast cancer progression. We compared Aurora A expression in tumor tissues in obesity breast cancer patients and a nonobesity cohort. We then analyzed the relationship between Aurora expression and clinical parameters and other biomarkers (Cyclin B1, Survivin, MMP11, Cathepsin L) of breast cancer and correlated them with survival (OS) and Recurrence-free survival (RFS). Results showed that patients with high Aurora A expression indicated a worse prognosis than obesity patients with low Aurora A expression, and the trend was non-significantly different in non-obesity patients. In addition, to further understand the Aurora A-related biological pathways involved in obesity breast cancer, Gene set enrichment analysis (GSEA) from TCGA was performed.

\section{Methods}

\section{Patients and Specimens}

We collected 706 surgical primary breast cancer specimens from the First Affiliated Hospital of China Medical University. All patients had undergone breast surgery between January 2011 and November 2016. The patients had not received any kind of neoadjuvant therapy before surgery, including radiotherapy, chemotherapy, endocrine therapy, target therapy, or immunotherapy. The patients with other malignant tumors were excluded. Finally, 517 patients were enrolled into analysis and the clinical stage was categorized by the 8 th AJCC TNM staging system. The status of ER, PR, HER2 and $\mathrm{Ki}-67$ were examined and reviewed in the hospital. All patients gave written informed consent for this study, which was approved by the regional ethics committee of China Medical University (approval number: 2016QL011). The study was conducted in accordance with the Declaration of Helsinki. An overview of histological and molecular characteristics of selected tumors is provided in Table 1.

\section{Assessments}

The available endpoint was recurrence-free survival (RFS), defined as the time from the date of surgery to
Table I Baseline Demographics and Characteristics for the Obesity and Non-Obesity Populations

\begin{tabular}{|c|c|c|c|c|}
\hline Characteristics & Total & $\begin{array}{l}\text { Obesity } \\
\text { BMI } \geq 25\end{array}$ & $\begin{array}{l}\text { Non- } \\
\text { Obesity } \\
\text { BMI<25 }\end{array}$ & $P$-value \\
\hline $\mathrm{N}$ & 517 & 198 & 319 & \\
\hline \multicolumn{5}{|l|}{ Sex } \\
\hline Female & 516 & 198 & 318 & \\
\hline Male & 1 & 0 & I & \\
\hline \multicolumn{5}{|l|}{ Age, years } \\
\hline Median & 52 & 53 & 51 & \\
\hline Range & $27-91$ & $28-78$ & $27-91$ & \\
\hline \multicolumn{5}{|l|}{ BMI, $\mathrm{kg} / \mathrm{m}^{2}$} \\
\hline Median & 24.0 & 27.3 & 22.8 & \\
\hline Range & $15.4-39.5$ & $25.0-39.5$ & $15.4-25.0$ & \\
\hline \multirow{3}{*}{$\begin{array}{l}\text { Menopausal Status } \\
\text { Premenopausal } \\
\text { Postmenopausal }\end{array}$} & & & & 0.027 \\
\hline & 242 & 80 & 162 & \\
\hline & 275 & 118 & 157 & \\
\hline \multirow{3}{*}{$\begin{array}{l}\text { Type of Surgery } \\
\text { Mastectomy } \\
\text { Breast-conserving }\end{array}$} & & & & 0.243 \\
\hline & 481 & 188 & 293 & \\
\hline & 36 & 10 & 26 & \\
\hline \multirow{3}{*}{$\begin{array}{l}\text { Tumor Size, cm } \\
\quad \leq 2 \\
>2\end{array}$} & & & & 0.001 \\
\hline & 133 & 35 & 98 & \\
\hline & 374 & 163 & 221 & \\
\hline \multirow{3}{*}{$\begin{array}{l}\text { Lymph Node } \\
\text { Positive } \\
\text { Negative }\end{array}$} & & & & 0.087 \\
\hline & 256 & 108 & 148 & \\
\hline & 261 & 90 & 171 & \\
\hline \multirow{3}{*}{$\begin{array}{l}\text { Tumor Grade } \\
\qquad \begin{array}{l}\text { I-2 } \\
3\end{array}\end{array}$} & & & & 0.154 \\
\hline & 463 & 172 & 291 & \\
\hline & 54 & 26 & 28 & \\
\hline \multirow{4}{*}{$\begin{array}{l}\text { Hormone } \\
\text { Receptor Status } \\
\text { ER or PR Positive } \\
\text { ER and PR Negative }\end{array}$} & & & & 0.647 \\
\hline & & & & \\
\hline & 374 & 146 & 228 & \\
\hline & 143 & 52 & 91 & \\
\hline \multirow{3}{*}{$\begin{array}{c}\text { HER2 Status } \\
\text { Positive } \\
\text { Negative }\end{array}$} & & & & 0.619 \\
\hline & 118 & 48 & 70 & \\
\hline & 399 & 150 & 249 & \\
\hline \multirow{2}{*}{$\begin{array}{l}\text { Adjuvant } \\
\text { Chemotherapy }\end{array}$} & & & & 0.096 \\
\hline & & & & \\
\hline Yes & 380 & 150 & 220 & \\
\hline No & 137 & 48 & 99 & \\
\hline
\end{tabular}

the date of the first local, regional or distant breast cancer relapse, distant metastases or any death before any type of relapse. Overall survival (OS) was defined as the time from surgery to the last follow-up date or death from any cause. Body Mass Index was calculated as body weight divided by the square of height. Our independent variable 
was BMI at baseline, categorized following $\mathrm{WHO}$ as overweight (BMI $\geq 25 \mathrm{~kg} / \mathrm{m}^{2}$, as the obesity cohort), and normal $\left(18.5 \leq \mathrm{BMI}<25 \mathrm{~kg} / \mathrm{m}^{2}\right.$, as the non-obesity cohort).

\section{Immunohistochemistry (IHC) for Aurora A Expression}

The immunohistochemical staining was performed on paraffin-embedded tissues according to the manufacturer's instructions of EnVision kit (MaiXin Biotech Co., Fuzhou, People's Republic of China). The deparaffinized sections were incubated in $\mathrm{H}_{2} \mathrm{O}_{2}$ (3\%) for 10 minutes, then blocked in $1 \%$ bovine serum albumin for 60 minutes. The primary antibody used was rabbit anti-human Aurora A monoclonal antibody (EP1008Y, ab52973, 1:150, Abcam, Cambridge, UK) incubated at $4{ }^{\circ} \mathrm{C}$ overnight and the secondary antibody used was goat anti-rabbit IgG H\&L (HRP, ab205718, 1:2000, Abcam, Cambridge, UK) at room temperature for 2 hours. These type-specific antibodies do not cross react with the other isoforms of Aurora kinases. ImageJ software was used to measure Aurora-A scoring. Aurora A expression on nucleus and cytoplasm were reviewed and confirmed, respectively. The immunohistochemical scoring principle was according to the staining intensity (no signal $=0$, weak $=1$, moderate $=2$, high $=3)$, and the percentage of staining cells $(0 \%=0,1 \%-$ $10 \%=1,11 \%-50 \%=2,51 \%-80 \%=3,81 \%-100 \%=4)$. The final score of 0-12 was based on multiplying the scores of intensity and percentage. Aurora A positive on nucleus or cytoplasm was confirmed only when the staining scores of Aurora $\mathrm{A} \geq 4$ and less than 4 was considered as negative/ weak. We tested different cutoffs with survival analysis to decide the optimal cutoff, when the statistical differences of recurrence-free survival (RFS) and overall survival (OS) were maximized in the additional files.

\section{Immunohistochemistry for Cyclin BI and Other Protein Expression}

Immunohistochemical analysis was also performed using monoclonal antibodies against Survivin (rabbit anti-human ab76424, 1:150, Abcam, Cambridge, UK), Cyclin B1 (rabbit anti-human, Y106, ab32053, 1:150, Abcam, Cambridge, UK), and polyclonal antibodies against MMP11 (rabbit antihuman ab53143, 1:150, Abcam, Cambridge, UK), Cathepsin L(rabbit anti-human ab203028, 1:150, Abcam, Cambridge, UK). A standard indirect immunoperoxidase procedure (ABC-Elite; Vector Laboratories, Burlingame, CA, USA) was used for all stains. In brief, primary antibodies were incubated at $4{ }^{\circ} \mathrm{C}$ overnight, followed by incubation with secondary antibody at room temperature for 60 minutes. Mayer's hematoxylin stain was used as a counterstain. The immunohistochemical staining was evaluated and categorized as positive and negative/weak. The expression status for ER, PgR, and HER-2 / neu was determined by immunohistochemical staining performed on full sections of tumors. ER or $\mathrm{PgR}$ was considered positive if $>10 \%$ of cells had positive nuclear staining. HER-2/neu expression status was categorized as negative ( 0 or $1+$, staining), $(2+$, membranous staining and negative by FISH), borderline ( $2+$, membranous staining and unknown by FISH), or overexpressed (3+, membranous staining and $2+$, membranous staining and positive by FISH).

\section{Analysis of Aurora A in the TCGA Public Database}

The breast cancer dataset, including mRNA expression and overall survival times, was obtained from TCGA (https://portal.gdc.cancer.gov/). The original data from TCGA was normalized and analyzed by the edgeR analysis method. Kaplan-Meier analysis of TCGA-BRCA data was used to analyze the 10-year overall survival of $\mathrm{BC}$ patients. Log rank test was used to calculate $P$ value. Additionally, online analysis database bc-GenExMiner v4.2 including two genomic datasets (http://bcgenex.centre gauducheau.fr/) was used to assess the association between Aurora A and survival of breast cancer patients.

\section{Gene Ontology Function Enrichment Analysis of Aurora A}

After normalizing the TCGA-BRCA dataset, we distinguished between Aurora A high group and Aurora A low group according to the mRNA expression level. The differences of GO functional enrichment between the two groups were obtained by Gene Set Variation Analysis (GSVA). GSEA was performed to annotate the Hallmark effector gene sets and the Kyoto Encyclopedia of Genes and Genomes (KEGG) signaling pathway associated with Aurora A mRNA expression in the TCGA-BRCA dataset. GSEA software was obtained from the Broad Institute (http://www.geo.org). Co-expressed genes of Aurora $\mathrm{A}$ in $\mathrm{BC}$ were analyzed from TCGA-BRCA expression data. Using Pearson correlation analysis, the correlation coefficient $\geq 0.2$ was used as the threshold to find the genes associated with higher levels of Aurora A. Protein-protein interact (PPI) network was constructed 
for Aurora A co-expressed genes using the STRING v11.0 database (https://string-db.org/), and the protein interaction score $\geq 0.2$, ie, medium and above reliability. To further analyze and screen the core genes in the PPI network.

\section{Statistical methods}

The SPSS software version 23.0 (IBM Inc., Armonk, NY, USA) was used for all analyses. The means of continuous numerical data were compared using a one-way analysis of variance with the Bonferroni correction. Differences between categorical variables were evaluated using the Pearson Chi-square test. Univariable associations between the events and parameters were plotted using the KaplanMeier method, and the cohorts were compared using the Log rank test. The Cox proportional hazard model was used to identify independent prognostic factors associated with recurrence-free survival and overall survival rates. All statistical tests were two-sided, and $P$ values $<0.05$ were considered to indicate statistical significance.

\section{Results}

\section{Patient Characteristics}

Between January 2011 and December 2016, 706 patients were screened and 517 patients were enrolled in this retrospective study. Of the 517 patients with early breast cancer, 198 cases $(38.3 \%)$ were diagnosed with high Body Mass Index $\left(\mathrm{BMI} \geqq 25 \mathrm{~kg} / \mathrm{m}^{2}\right.$, defined as the obesity cohort), and $319(61.7 \%)$ with normal BMI (less than $25 \mathrm{~kg} / \mathrm{m}^{2}$ ) were put in the non-obesity cohort. Baseline characteristics of the study population are described in Table 1 . The median BMI was $27.3 \mathrm{~kg} / \mathrm{m}^{2}$ (range: $25.039 .5 \mathrm{~kg} / \mathrm{m}^{2}$ ) in the obesity cohort and $22.8 \mathrm{~kg} / \mathrm{m}^{2}$ (range: $15.424 .9 \mathrm{~kg} / \mathrm{m}^{2}$ ) in the nonobesity cohort. Only one male patient was present in the non-obesity cohort and the other patients were all female in both the obesity and non-obesity cohorts.

Most baseline characteristics were well balanced between the obesity and non-obesity groups. The mean age at diagnosis was 53 years (range: 2878 years) in the obesity cohort and 51 years (range: 2791 years) in the nonobesity cohort. There was no difference in the type of surgery, tumor grade, hormone receptor status or HER2 status between the obesity and non-obesity cohorts. According to menopausal status, obesity was positively associated with more postmenopausal patients [obesity cohort vs non-obesity cohort: $59.6 \%$ (118/198) vs $49.2 \%$ (157/319), $P=0.027$ ], larger tumor size [obesity cohort vs non-obesity cohort: $82.3 \%$ (163/198) vs 69.3\% (221/319),
$P=0.001]$. Interestingly, the incidence of lymph node metastatic tended to be higher in patients with high BMI in the obesity cohort than in those in the non-obesity cohort; However, patients who received adjuvant chemotherapy (including chemotherapy, anti-HER2 target therapy) were more common in the obesity cohort than in those in the non-obesity cohort, as shown in Table 1. Our findings demonstrated that obesity was also associated with aggressive tumor characteristics (larger tumor size, more lymph node-positive). The association between BMI and breast cancer molecular subtypes is shown in Figure 1. The molecular subtypes distribution was similar between the obesity and non-obesity cohorts, approximately $10 \%$ was HER2positive breast cancer and approximately $75 \%$ was in Luminal breast cancer subtype. More triple-negative breast cancer was not indicated in our study, it was an inverse finding compared with a previous study (only 15\% triplenegative breast cancer in our cohort vs $33.3 \%$ and $40.0 \%$ in overweight and obesity premenopausal patients in triplenegative as presented by Chen et al). ${ }^{14}$

\section{Aurora A Expression was Associated with Clinical Characteristics}

In all 517 tested samples, 240 (46.4\%) patients had positive cytoplasm Aurora A expression ( $\geqq 4$ cytoplasm staining score) and 239 (45.2\%) had nucleus Aurora A-positive ( $\geqq 4$ nucleus staining score) (Figure 2). Moreover, we observed that the localization of Aurora A is scattered from cytoplasm to nucleolus. However, the incidence of nucleus Aurora A-positive and cytoplasm Aurora A-positive were approximately $46 \%$ in our study. Given the similar positive ratio of Aurora A expression in cytoplasm and nucleus, only cytoplasm Aurora A expression was explored in the following investigations.

We validated Aurora A over expression using an independent cohort with a total of 517 breast carcinoma tissues (319 non-obesity patients and 198 obesity patients). The expression level of Aurora A-positive (staining scores $\geqq 4$ ) was significantly higher in obesity breast carcinoma compared with non-obesity cancer carcinoma (54.6\% vs $40.5 \%$, $\left.\chi^{2}=9.79, P=0.002\right)$. In addition, the Aurora A high staining (staining scores $\geqq 7$ ) in the obesity cohort were obviously higher than those of Aurora $\mathrm{A}$ in the non-obesity cohort with a statistical difference $(17.7 \%$ vs $8.5 \%, P$ value $=0.002$, Figure 3 ). In the immunohistochemistry assay, the morphological and statistical results confirmed that the expression level of Aurora A-positive was significantly associated with 


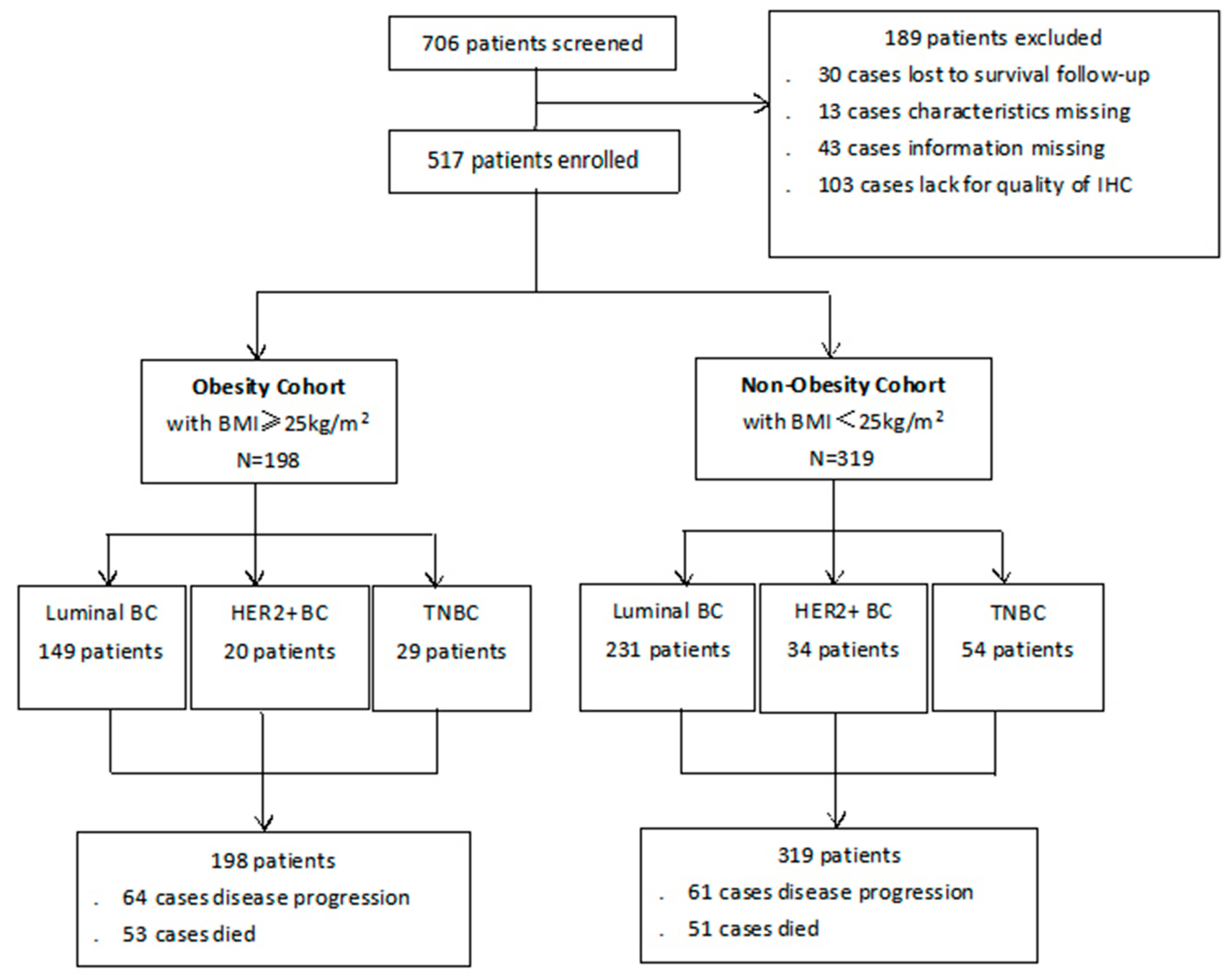

Figure I Patient disposition in our study.

Note: Flow diagram showing patient enrollment, allocation, follow-up, and analysis in this study.

Abbreviations: BMI, body mass index; $\mathrm{BC}$, breast cancer.

hormone receptor status $(68.4 \%$ vs $77.9 \%, P=0.015)$ and HER2 status $(28.7 \%$ vs $17.9 \%, P=0.003)$. Cytoplasm Aurora A-positive tumors had larger tumor size, though not statistically significant $(78.1 \%$ vs $71.1 \%, P=0.070)$. The distributions of other clinical characteristics, including age, menopausal status, grade and lymph node metastases, were even when grouped with Aurora A in both groups ( $P=0.876$, $P=0.881, P=0.349, P=0.677$, respectively).

\section{Association of High Aurora A Expression with Poor OS and RFS in the Obesity Cohort}

To explore whether Aurora A expression levels will affect the clinical outcomes in both cohorts, we constructed a prognostic classifier using Kaplan-Meier analysis from 517 patients in our study. As shown in Figure 4, Aurora A expression (staining scores $\geqq 4$ ) was remarkably and significantly associated with OS (OR=1.76, 95\% CI: 1.03 3.02, $P=0.041)$ among obesityrelated breast carcinoma patients. The 8 -year OS ratio in the Aurora A low expression group was $81.1 \%$ whereas in the high expression group it was $69.5 \%$ in the obesity cohort. Interestingly, higher expression of Aurora A was not associated with a shorter overall survival time among the non-obesity breast cancer patients $(\mathrm{OR}=1.40,95 \%$ CI: 0.79 2.45, $P=0.229$ ). The 8 -year OS ratio was $85.8 \%$ months in the low Aurora-A expression group and $81.4 \%$ in the high Aurora-A expression group in the non-obesity cohort. To investigate the optimal cut-off value for Aurora A on prognosis, high Aurora A expression (staining scores $\geq 1$ ) was not associated with OS 

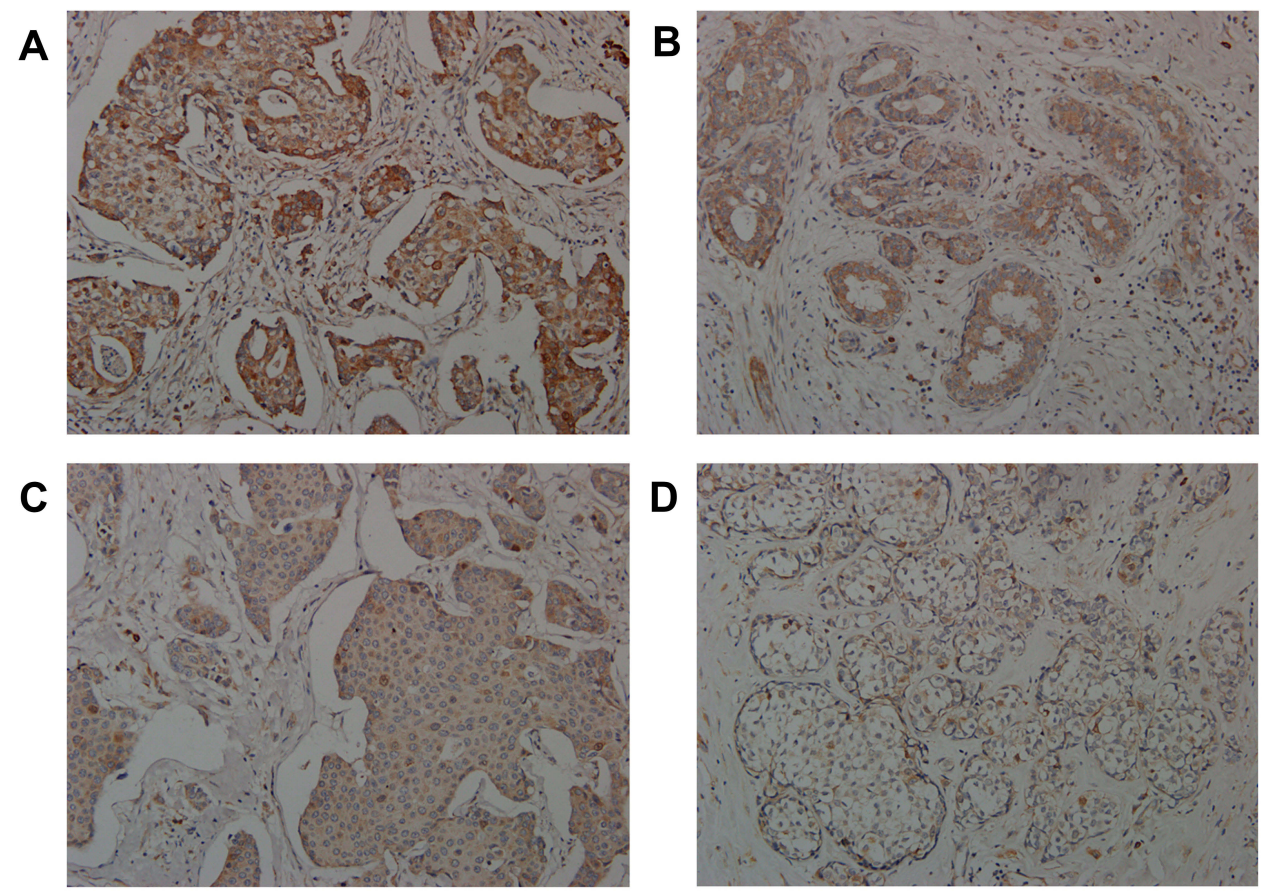

Figure 2 Representative micrographs of immunohistochemical stains showing Aurora A expression high staining (A), Aurora A expression median staining (B), Aurora A expression weak staining (C), Aurora A expression negative (D).

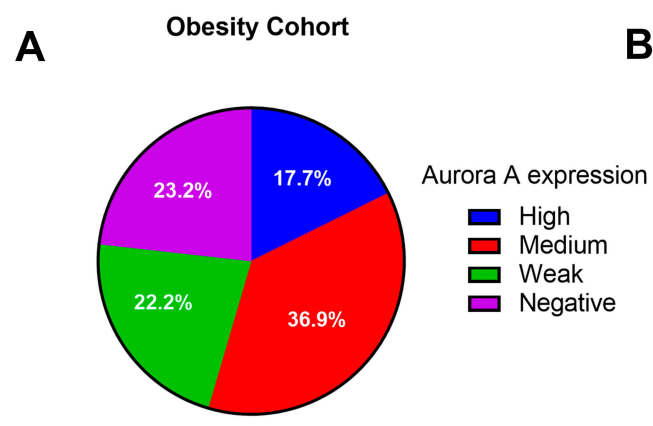

$\mathbf{N}=198$
B

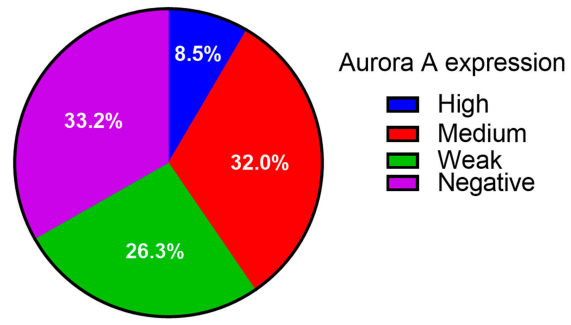

$\mathbf{N}=319$

Figure 3 Association of Aurora A expression with BMI in the obesity cohort (A), and the non-obesity cohort (B).

(total cohort: $\mathrm{OR}=1.356,95 \% \mathrm{CI}: 0.873 \sim 2.107, P=0.175$; obesity cohort: $\mathrm{OR}=1.112,95 \% \mathrm{CI}: 0.593 \sim 2.085, P=0.741$; non-obesity cohort: $\mathrm{OR}=1.430,95 \% \quad \mathrm{CI}: \quad 0.773 \sim 2.645$, $P=0.255$ ). Much higher Aurora A expression (staining scores $\geq 7$ ) was remarkably but non-significantly associated with OS (total cohort: $\mathrm{OR}=2.059,95 \% \mathrm{CI}$ : $0.889 \sim 4.853, P=0.084$; obesity cohort: $\mathrm{OR}=2.269,95 \% \mathrm{CI}: 0.959 \sim 5.314, P=0.079$; non-obesity cohort: $\mathrm{OR}=1.766,95 \%$ CI: $0.429 \sim 7.264$, $P=0.431$ ). Thus, the cut-off value was defined as high Aurora A expression with 4 and more in the following investigation.

As for RFS in Figure 5, the expression levels of Aurora A expression genes have no significance with RFS statistically in non-obesity and obesity patients. The 5-year RFS ratio in the Aurora A low expression group was $83.2 \%$ and $82.2 \%$ and in the high Aurora $\mathrm{A}$ expression in nonobesity cohort $(\mathrm{OR}=1.29,95 \% \mathrm{CI}: 0.77 \sim 2.16, P=0.307)$. While 5-year RFS ratio in the high Aurora A expression group $(71.3 \%)$ was a little shorter than the 5-year RFS ratio in the low Aurora A expression group $(\mathrm{OR}=1.58,95 \% \mathrm{CI}$ : $0.96 \sim 2.57, P=0.072$ ) in the obesity cohort.

With respect to RFS and OS, it was shown that maybe there are many factors affecting survival in addition to the level of Aurora A expression. We performed univariate and multivariate analysis with the logistic regression model. 

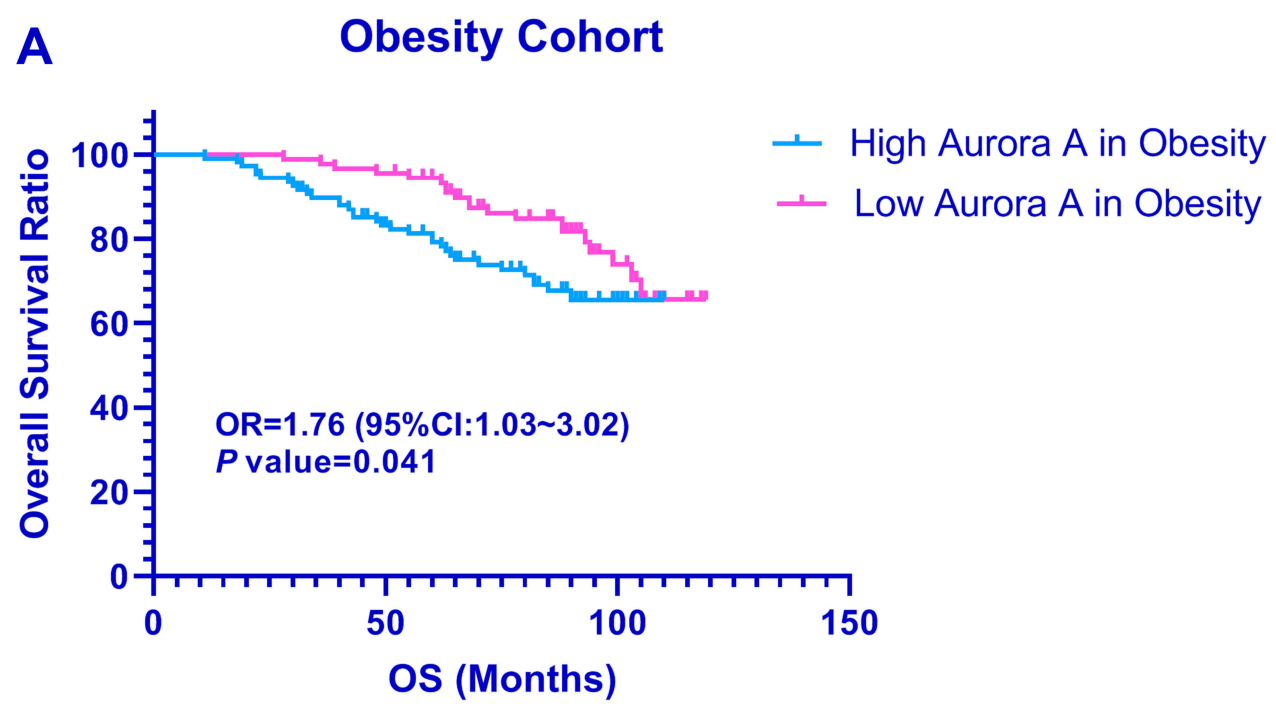

B Non-Obesity Cohort

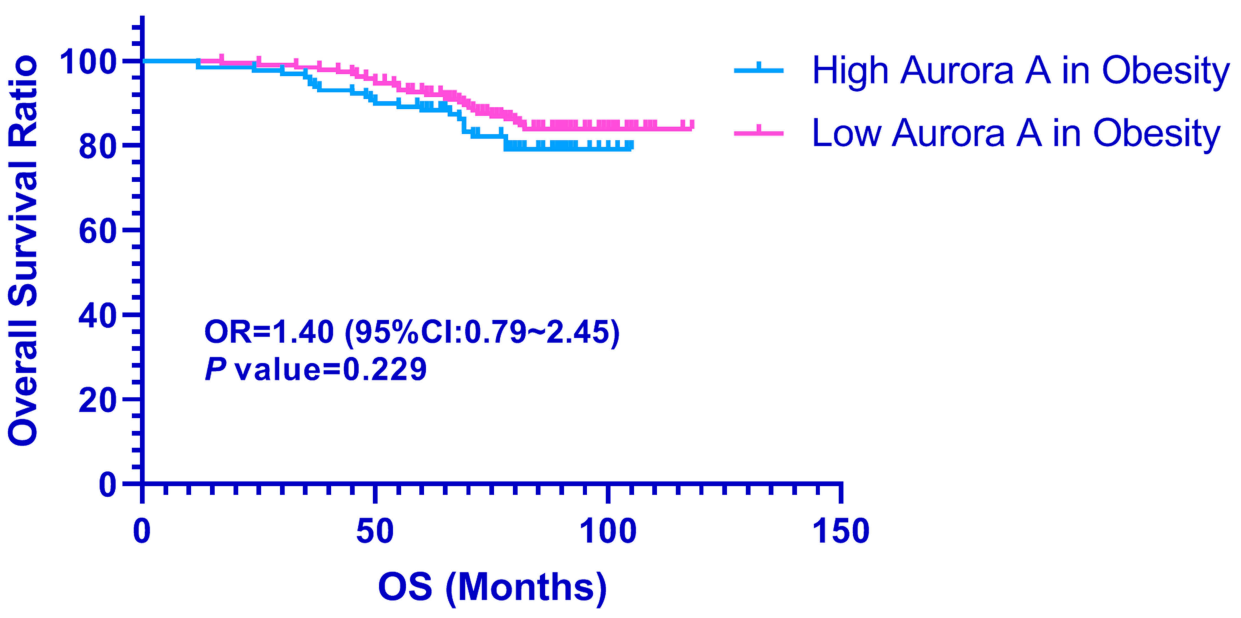

Figure 4 Prognostic significance of Aurora A expression for OS in breast cancer.

Note: Kaplan-Meier survival curves for overall survival as a function of expression of Aurora A in patients with obesity breast cancer (A), and non-obesity breast cancer (B).

After adjusting for age, tumor size, lymph node status, HR status, HER2 status, adjuvant chemotherapy and Aurora A staining score, the OR for Aurora A expression in the obesity cohort was $5.318(95 \% \mathrm{CI}, 1.298-21.785 ; P=0.02)$ while the OR was $5.318(95 \% \mathrm{CI}, 1.298-21.785 ; P=0.02)$ for Aurora $\mathrm{A}$ in the non-obesity cohort. Tumor size, adjuvant chemotherapy, menopausal status, ER status and PR status were not significant factors for overall survival among patients with early breast cancer (Table 2). Lymph node metastases, age, HER2-positive, high BMI and high Aurora A staining score disease were poor prognostic factors for OS in univariate analysis. In multivariate analysis, Aurora A and lymph node metastases were significantly poor prognostic factors for OS, and borderline significance was noted for high BMI. To explore the interaction between Aurora A expression and BMI on overall survival, the interaction 


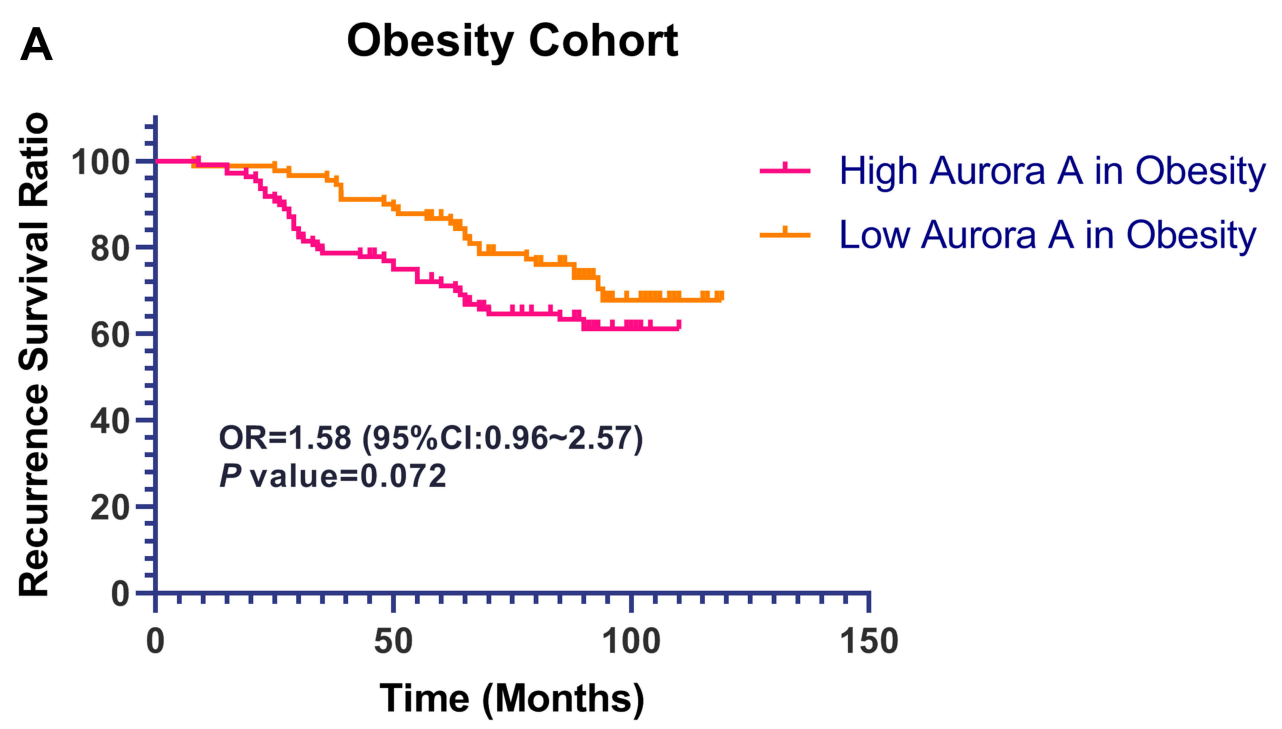

B Non-Obesity Cohort

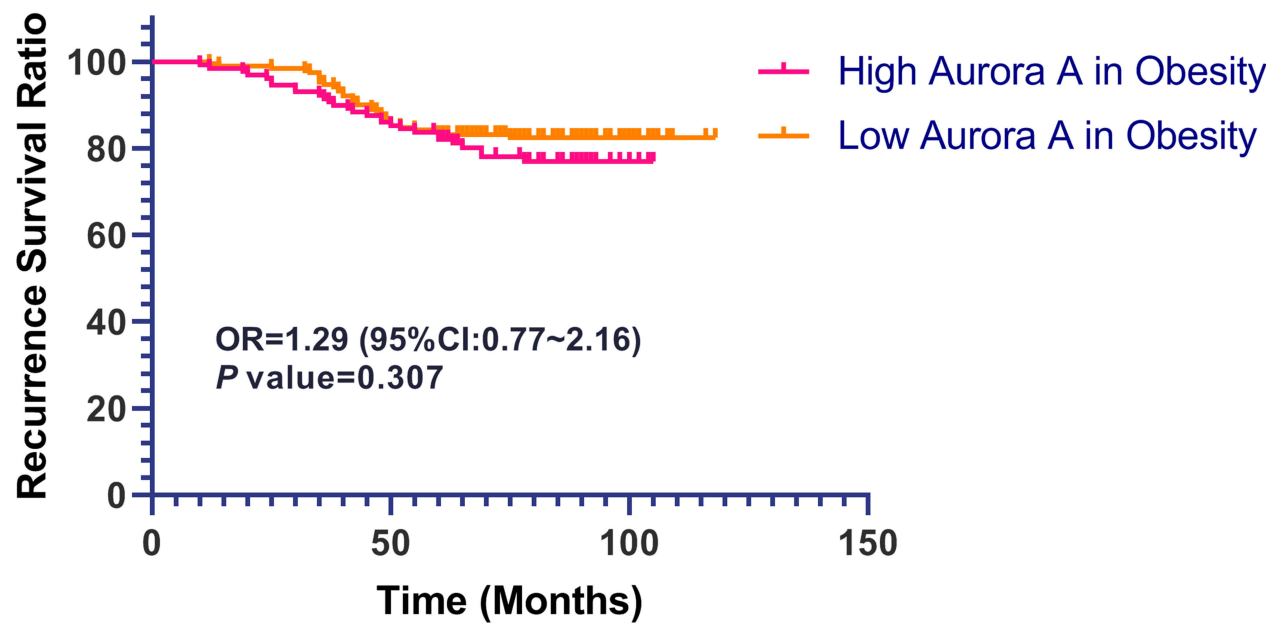

Figure 5 Prognostic significance of Aurora A expression for RFS in breast cancer.

Note: Kaplan-Meier survival curves for relapse-free survival as a function of expression of Aurora $A$ in patients with obesity breast cancer (A), and non-obesity breast cancer (B).

$P$ value was 0.021 for Aurora A interacting with BMI in the prognosis prediction $(\mathrm{OR}=1.586,95 \% \mathrm{CI}=1.073 \sim 2.343)$.

\section{Prognostic Role of Aurora A Expression and Functional Enrichment in Breast Cancer from TCGA}

To further understand and confirm the prognostic role of Aurora A expression in breast cancer, all breast cancer patients from the TCGA database (two GSE: GSE3494 for survival analysis; GSE78958 for obesity-related gene analysis) $)^{19,20}$ were categorized according to the median Aurora A expression value (high Aurora A expression group and low Aurora A expression group). Patients who lacked complete clinical data (including overall survival and OS status) were excluded from the analysis. To explore the potential mechanism of Aurora A and its related signal pathway in breast cancer, cytoplasm Aurora A expression was examined in our investigation and Aurora A expression in cytoplasm and nucleus from 
Table 2 Univariate and Multivariate Cox Regression Analyses for OS in Our Study

\begin{tabular}{|c|c|c|c|c|}
\hline \multirow{2}{*}{$\frac{\text { Variables }}{\text { Overall Survival }}$} & \multicolumn{2}{|l|}{ Univariate Analysis } & \multicolumn{2}{|c|}{ Multivariate Analysis } \\
\hline & HR (95\% Cl) & $P$-value & HR (95\% Cl) & $P$-value \\
\hline $\begin{array}{l}\text { Age } \\
<60 \text { vs } \geq 60\end{array}$ & $0.502(0.276-0.9 \mid 4)$ & 0.024 & $0.652(0.377-1.127)$ & 0.125 \\
\hline $\begin{array}{l}\text { Menopausal status } \\
\text { Pre- vs Post-menopausal }\end{array}$ & $1.094(0.747-1.604)$ & 0.644 & & \\
\hline $\begin{array}{l}\text { Tumor size } \\
\text { TI vs } \mathrm{T} 2 / \mathrm{T} 3 / \mathrm{T} 4\end{array}$ & $1.088(0.694-1.706)$ & 0.713 & & \\
\hline $\begin{array}{l}\text { Lymph node } \\
\text { Positive vs Negative }\end{array}$ & $1.724(1.029-2.403)$ & 0.002 & $1.610(1.377-2.507)$ & 0.019 \\
\hline $\begin{array}{l}\text { Adjuvant chemotherapy } \\
\text { Yes vs No }\end{array}$ & $1.205(0.815-1.780)$ & 0.350 & & \\
\hline $\begin{array}{l}\text { BMI } \\
<25 \text { vs } \geq 25\end{array}$ & $1.651(1.123-2.428)$ & 0.011 & $1.470(0.998-2.502)$ & 0.053 \\
\hline $\begin{array}{l}\text { ER status } \\
\text { Positive vs Negative }\end{array}$ & $1.160(0.770-1.747)$ & 0.479 & & \\
\hline $\begin{array}{l}\text { PR status } \\
\text { Positive vs Negative }\end{array}$ & $0.966(0.652-1.432)$ & 0.863 & & \\
\hline $\begin{array}{l}\text { HER2 status } \\
\text { Positive vs Negative }\end{array}$ & $1.320(0.918-1.988)$ & 0.096 & $1.358(0.817-2.256)$ & 0.238 \\
\hline $\begin{array}{l}\text { Aurora } A \text { staining score } \\
<4 \text { vs } \geq 4\end{array}$ & $1.669(1.132-2.459)$ & 0.010 & $1.629(1.091-2.439)$ & 0.017 \\
\hline
\end{tabular}

TCGA data were included (Figure 6A). The difference of Aurora A expression in Pan-cancer is indicated in Figure 6B. Functional enrichment and protein-protein interact (PPI) network were analyzed with $\mathrm{R}$ packages from the TCGA database. The STRING database identified interaction relationships in 105 out of the 185 methylationrelated Aurora A (68 downregulated and 37 upregulated). The 10 interaction relationship pairs among the Aurora A were used to construct a PPI network including IGFR1/PKA and $\mathrm{PLC} / \mathrm{Ca}^{2+}$ signal pathways (Figure 6C). Two human oncogenes, CCNB1 and CDK1, were indicated as hub genes of the PPI network as they were shared and ranked in the top 15 for four topological characteristics. Subsequently, four highly connected PPI sub-modules (Figure 6D) were extracted from the overall PPI network using MCODE. BinGO enrichment analysis demonstrated that the genes in module (MCODE score=5.982) were involved in the mitotic cell cycle (AURKA, CDK1 and CCNB1). Kaplan-Meier survival analysis indicated that the high Aurora A expression group had worse prognosis compared with the low Aurora A expression group $(\mathrm{N}=236, \quad \mathrm{HR}=1.47, \quad 95 \% \quad \mathrm{CI}: \quad 1.14-1.90, \quad P=0.003)$ (Figure 6E). Obesity-related gene analysis was conducted from GSE78958. The 121 downregulated/hypermethylated and 61 upregulated/hypomethylated Aurora
A-related genes were respectively uploaded to DAVID to predict their functions. Using the threshold value of FDR $<0.05,18 \mathrm{GO}$ biological process terms were obtained for the downregulated/hypermethylated Aurora A, including "cell cycle" and "inflammatory response" (FOS).

\section{Correlation Between Aurora A and Other Proliferation and Invasion Biomarkers}

We used the Spearman correlation test to describe the connection between the level of Aurora A and other proliferation and invasion biomarkers (proliferation biomarkers: Survivin, Cyclin B1; Invasion biomarkers: MMP11 and Cathepsin L, Figure S1) in the obesity cohort. All obesity patients with breast cancer were included in this analysis. The IHC scores was evaluated as grade variables. We found that Aurora A level on tumor cytoplasm had broad connections with other proliferation biomarkers including Cyclin B1 (correlation coefficient $=0.227$, $P=0.001$ ) and MMP11 (correlation coefficient $=0.169$, $P=0.017$ ). Aurora A levels did not have a positive relation with Cathepsin L (correlation coefficient $=0.051$, $P=0.483$ ), and Survivin (correlation coefficient $=0.043$, $P=0.547$ ) (Table 3). 

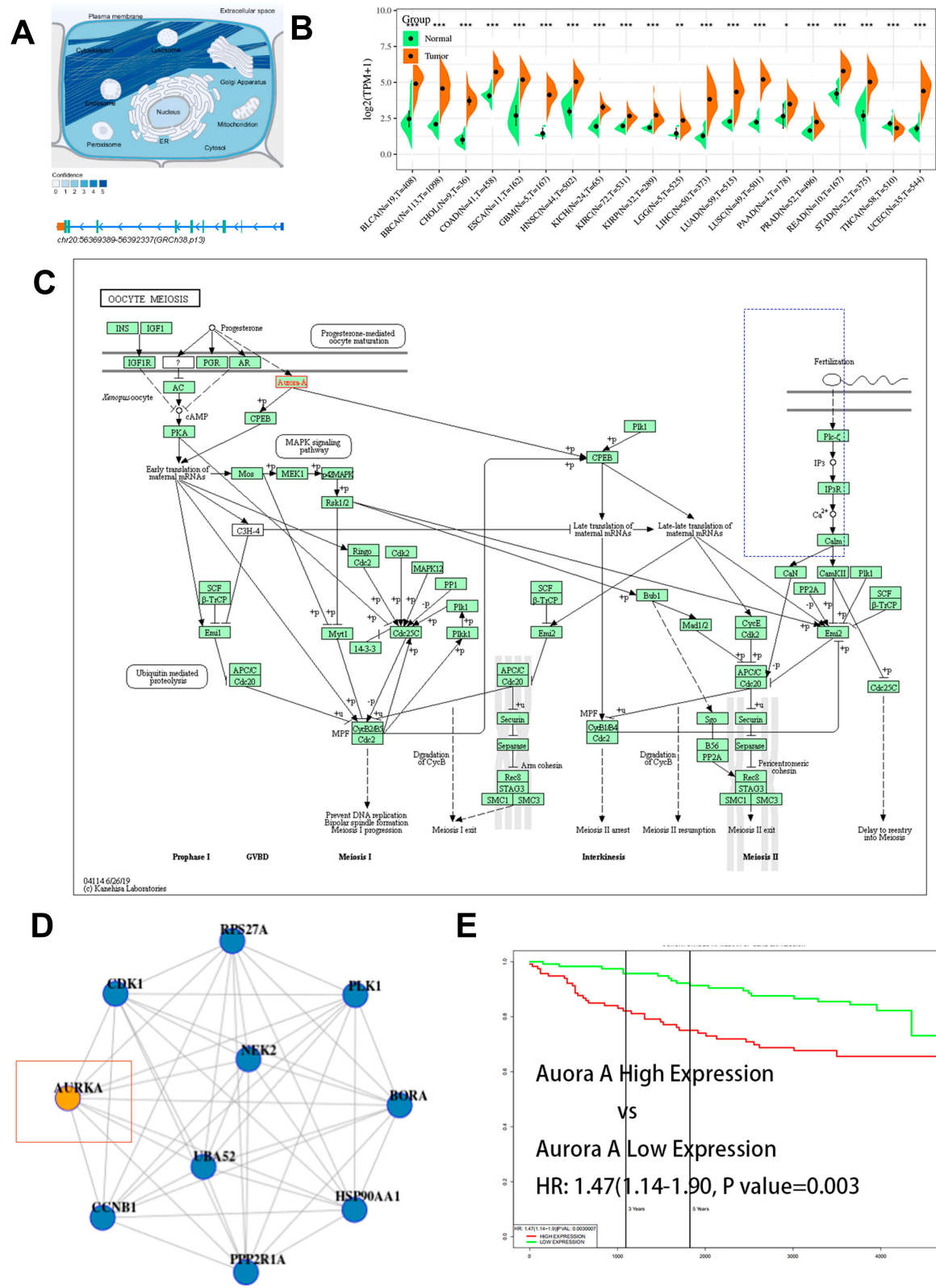

E

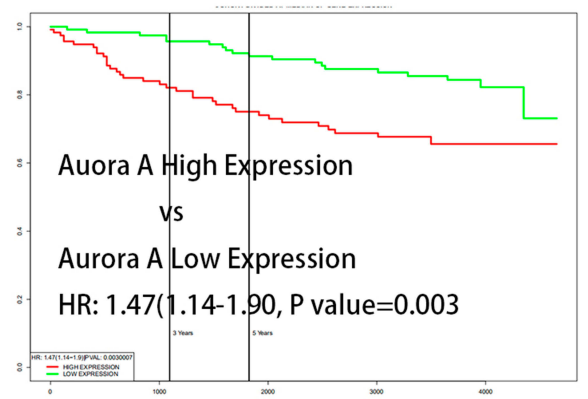

Figure 6 Prognostic significance of expression of Aurora A in breast cancer from TCGA public database.

Notes: Expression of Aurora A in cellular structure (A), the significant differences between normal and cancer in Pan-cancer (B). Construction of the PPI network and identification of a significant module with high Aurora A expression by cytoscape (C). The most significant module (AURKA and CCNBI) was obtained from the PPI network using molecular complex detection (D). Kaplan-Meier survival curves for overall survival as a function of expression of Aurora $A$ in patients with breast cancer (E). Abbreviations: N, normal; T, tumor; BLCA, bladder urothelial carcinoma; BRCA, breast invasion carcinoma; CHOL, cholangiocarcinoma; COAD, colon adenocarcinoma; ESCA, esophageal carcinoma; GBM, glioblastoma multiforme; HNSC, head and neck squamous cell carcinoma; KICH, kidney chromophobe; KIRC, kidney renal clear cell carcinoma; KIRP, kidney renal papillary cell carcinoma; LGG, brain lower grade glioma; LIHC, liver hepatocellular carcinoma; LUAD, lung adenocarcinoma; LUSC, lung squamous cell carcinoma; PAAD, pancreatic adenocarcinoma; PRAD, prostate adenocarcinoma; READ, rectum adenocarcinoma; STAD, stomach adenocarcinoma; THCA, thyroid carcinoma; UCEC, uterine corpus endometrial carcinoma.

\section{Discussion}

Although Aurora A has been intensively studied in series of human malignancies as a carcinogenic factor, ${ }^{21-23}$ the impact of Aurora A on the malignant behavior among obesity breast cancer remains elusive. Consistent with previous studies, ${ }^{16}$ our study also indicated a poor prognosis biomarker in breast cancer and its expression was correlated to high malignant aggressiveness. In particular, we found that, for the first time, Aurora A expression was tightly associated with poor survival in obesity patients with early breast cancer, and the phenomenon did not appear in the non-obesity cohort. These 
Table 3 Correlation Between Aurora A Expression and Other Biomarkers by Spearman Coefficient Analysis in Obesity Patients with Early Breast Cancer

\begin{tabular}{|l|l|l|l|l|}
\hline Aurora A vs. & Cyclin B I & MMP I I & Cathepsin L & Survivin \\
\hline Coefficient value & 0.227 & 0.169 & 0.051 & 0.043 \\
P-value & 0.001 & 0.017 & 0.483 & 0.547 \\
\hline
\end{tabular}

results highlight that Aurora A plays a crucial role during obesity-related poor differentiation and as a mechanism of relapse and distant metastases. More importantly, elevated expression of Aurora A was validated and has been linked to worse clinical outcomes in early breast cancer from GEO database and identified by a panel of 24 DEGs. Functional enrichment and PPI network analysis stressed that cell cycle genes were closely related to carcinogenesis of obesity related breast cancer. Cyclin B1 and MMP11 were tightly connected with Aurora A in our obesity tissue IHC correlation test. Our results demonstrated Aurora A could be a potential promising therapeutic target in improving clinical outcomes in obesity breast cancer.

Firstly, we validated Aurora A over expression using an independent cohort with a total of 517 breast carcinoma tissues (319 non-obesity patients and 198 obesity patients). We observed that the localization of Aurora A is scattered from cytoplasm to nucleolus. However, the incidence of cytoplasm Aurora A-positive and nucleus Aurora A-positive were approximately $46 \%$ in our study. Given the similar positive ratios of Aurora A expression in cytoplasm and nucleus, only cytoplasm Aurora A expression was explored in this study. Obesity is an increasing society burden worldwide including in the People's Republic of China, ${ }^{3}$ thus this study focused on investigating Aurora A expression in obesity compared with non-obesity patients. Interestingly, higher expression of Aurora A was not associated with a shorter overall survival time among non-obesity breast cancer. Moreover, high Aurora A expression was remarkably associated with OS (the absolute 8-year OS ratio difference was: $11.6 \%$ ) in the obesity cohort.

The molecular mechanisms that link obesity breast cancer are complex, the main mechanisms which can explain this link are leptin, adipose chronic inflammation, sex hormone alternation, and insulin signaling. ${ }^{4,24-26} \mathrm{Kim}$ et $\mathrm{al}^{27}$ demonstrated Aurora A-related cell cyclin with a spatially localized response to IGF-1 using high throughput single-cell imaging to confirm the hypothesis that the presence of various cellular sub-populations interact with IGF-1. Previous studies highlighted that high Aurora A expression was strongly associated with worse survival of breast cancer and as an independent prognostic marker. ${ }^{16,21}$ In particular, among TNBC patients with high level of Aurora A expression, the peak time of distant recurrence risk was at the first 3 years and declined rapidly thereafter, whereas patients with low Aurora A expression showed a relatively constant risk of recurrence during the entire follow-up period, ${ }^{16}$ it indicated that $\mathrm{TNBC}$ with high Aurora A expression may be the basal-like intrinsic subtype by PAM50 analysis and TNBC with low Aurora A expression was luminal subtype. The underlying mechanism of Aurora A in triple-negative breast cancer requires further investigation. In our study, Aurora A expression was found even among breast cancer subtypes between the obesity and nonobesity cohorts. Aurora A has been reported to phosphorylate several crucial proteins involved in cell cycle checkpoint after DNA damage and diminished transcriptional activity, causing genetic instability and ultimately increasing cancer development and disease progression. ${ }^{28}$ Interestingly, polymorphisms in AURKA (Aurora A gene, rs6099128 GG genotype) involved in chemotherapy and endocrine therapy resistance and decreased clinical outcome in breast cancer and was confirmed in taxane-resistance breast cancer cell lines. ${ }^{29,30}$ Studies of antiestrogens resistance breast cancer cell culture models by mimicking ER disclosed that Aurora A was increased and mediated cell proliferation and cell cycle in both antiestrogen and aromatase inhibitor resistant breast cancer cells. ${ }^{31-33}$ Comprehensive analyses with a library of 195 kinase inhibitors identified Aurora A inhibitor JNJ-7706621 as a preferential inhibitor of endocrine therapy resistant breast cancer cell lines. ${ }^{34}$ Resensitivity to tamoxifen treatment was recovered with Aurora A siRNA or Aurora A inhibitor, indicating a vital role for Aurora $\mathrm{A}$ in endocrine therapy resistance. ${ }^{17,31}$

Considering the role of Aurora A in duplicating centrosomes from the beginning of S phase, shifting to the bipolar spindle microtubules during mitosis, and, finally, moving to perinuclear materials of the daughter cells at the end of mitosis, ${ }^{33}$ we hypothesize that overexpression of Aurora A was closely correlated with cell cyclin-related protein (including Cyclin B1) in obesity patients. Cell proliferation is closely associated with the cell cycle, which is rigidly regulated by a series of kinases, among which Cyclin B1 and Aurora A are two important substrates involved in the promotion of $\mathrm{G} 1 / \mathrm{S}$ and $\mathrm{G} 2 / \mathrm{M}$ transition, respectively. Previous studies have implicated the role of Cyclin B1 in the regulation of cell proliferation in breast cancer. ${ }^{35-37}$ In 
our study, the results indicated that Aurora A-Cyclin B1 axis plays a crucial role in obesity breast cancer.

The degree of heterogeneity and the expression pattern of biomarkers under study may constitute a potential problem in tissue microarray-based study. However, representativity of tissue microarray-based studies has been carefully examined, and the data was identified by high-throughput tissue microarray screening analysis generally corresponding well with those identified using full-size tissue sections. Further to validate Aurora A expression in obesity patients with breast cancer and its potential mechanism, comprehensive analysis from GEO database and PPI network was used by MCODE model. However, it is a puzzle that the identified Aurora A in our training cohorts was only in obesity patients with early breast cancer and could not easily be validated in external TCGA cohorts. ${ }^{19,20}$ One reason might be bias of BMI information from GEO database, BMI information was not a required item while submitting to the genomics database. Another reason was the effects of Aurora A have broad confidence intervals so that it is difficult to identify using a single validation database. To address these issues, it is necessary to validate the signature of Aurora A expression in a larger obesity sample and prospective investigation. Serial studies have already confirmed that Aurora A inhibition can induce programmed cell death and cellular senescence via cell cycle axis in vitro and in vivo. Pharmacological inhibition of Aurora A improved sensitivity to PAK1 inhibition and antiestrogens treatment in p21-activated or RB1 loss breast cancer cell lines. ${ }^{38-40}$ RB1 loss was a potential mechanism to $\mathrm{CDK} 4 / 6$ inhibitors in clinical situations, and limited the application of CDK4/6 inhibitors, causing more benefit for abundance HR-positive breast cancer patients. $^{41}$ Thus, we further explored the effects of Aurora A inhibition by assessing the cell survival, cell morphology changes and effect on CDK4/6 inhibitorsrelated resistance in breast cancer cell lines.

Limitations of our study are obvious. First, although the known IHC microarray and TCGA sequencing data were included to confirm the expression of crucial protein Aurora A was linked to poor prognosis in obesity breast cancer, the sample size associated with obesity breast cancer was small. Therefore, more clinical samples need to be collected to further confirm Aurora A expression level. Second, this was an exploratory, hypothesis-generating retrospective study with a rather small and heterogeneous cohort.
Especially when the cut-off value of BMI definition was inconsistent with WHO criterion, ${ }^{3}$ the inclusion in the obesity cohort was overweight/obesity. Due to the strict criteria, obesity (BMI $\geqq 30 \mathrm{~kg} / \mathrm{m}^{2}$ ) patients only accounted for $5.6 \%$ of the total (29/517), we had to admit that the data of strict obesity patients was insufficient. However, overweight is as important as obesity in receiving increasing attention in society and medicine. Second, without a settled cutoff for Aurora A, we ran the survival analysis repeatedly with different cutoffs in our samples and TCGA data and the optimized cutoffs was decided in large cohort when the survival difference between groups maximized. Third, the lack of clinical information like BMI data in the GSE database limited the depth of this research. Last but not least, the optimal cut-off value for Aurora A on prognosis, much higher Aurora A expression (staining scores $\geq 7$ ) was remarkably but non-significantly associated with OS and the optimal cut-off value will be investigated in further sample studies. For further investigation, a prospective study with a larger cohort is needed and more elaborate experiment design (including Aurora A expression/inhibition and CDK4/6 inhibitors-resistance) may be expected.

\section{Conclusions}

In summary, we demonstrate here for the first time that high expression of Aurora A was notably correlated with early recurrence and poor overall survival in obesity patients with early breast cancer. The ongoing development of targeting Aurora A-Cyclin B1 axis signaling mechanisms will open new promising approaches for obesity breast cancer prevention and avoiding treatment resistance.

\section{Abbreviations}

BMI, body mass index; CI, confidence interval; ER, estrogen receptor; HER2, human epidermal growth factor receptor 2; OR, odd ratio; mo, month; OS, overall survival; RFS, recurrence-free survival; PgR, progesterone receptor; WHO, World Health Organization.

\section{Acknowledgment}

We thank Prof. Zheng $\mathrm{H}$ for association for IHC detection and guideline in this study.

\section{Funding}

This work was supported by the Natural Science Foundation of Liaoning Province (No. 20180551215), Key R \& D 
guidance plan of Liaoning Province (2019JH8/10300020), Key Research Project of Liaoning (grant number 2018225076), CSCO Project (Y-2019Genecast-019, YHR2018-362), Wujieping Project (320675018541) and LiaoNing Revitalization Talents Program (XLYC1907160).

\section{Disclosure}

The authors report no conflicts of interest in this work.

\section{References}

1. DeSantis CE, Ma J, Gaudet MM, et al. Breast cancer statistics, 2019. CA Cancer J Clin. 2019;69(6):438-451. doi:10.3322/caac.21583

2. Aleskandarany MA, Vandenberghe ME, Marchiò C, et al. Tumour heterogeneity of breast cancer: from morphology to personalised medicine. Pathobiology. 2018;85(1-2):23-34. doi:10.1159/000477851

3. Chu DT, Phuong TNT, Tien NLB, et al. The effects of adipocytes on the regulation of breast cancer in the tumor microenvironment: an update. Cells. 2019;8(8):E857. doi:10.3390/cells8080857

4. Atoum MF, Alzoughool F, Al-Hourani H. Linkage between obesity leptin and breast cancer. Breast Cancer (Auckl). 2020;14:11782 23419898458.

5. Lartey ST, Si L, Otahal P, et al. Annual transition probabilities of overweight and obesity in older adults: evidence from World Health Organization study on global AGEing and adult health. Soc Sci Med. 2020;247:112821. doi:10.1016/j.socscimed.2020.112821

6. Ayoub NM, Yaghan RJ, Abdo NM, et al. Impact of obesity on clinicopathologic characteristics and disease prognosis in pre- and postmenopausal breast cancer patients: a retrospective institutional study. J Obes. 2019;2019:3820759. doi:10.1155/2019/3820759

7. Zimta AA, Tigu AB, Muntean M, Cenariu D, Slaby O, BerindanNeagoe I. Molecular links between central obesity and breast cancer. Int J Mol Sci. 2019;20(21):5364. doi:10.3390/ijms20215364

8. Chan DS, Vieira AR, Aune D, et al. Body mass index and survival in women with breast cancer-systematic literature review and meta-analysis of 82 follow-up studies. Cancer Prev Res (Phila). 2012;5(4):583-592. doi:10.1158/1940-6207.CAPR-11-0482

9. Cecchini RS, Costantino JP, Cauley JA, et al. Body mass index and the risk for developing invasive breast cancer among high-risk women in NSABP P-1 and STAR breast cancer prevention trials. Ann Oncol. 2014;25(10):1901-1914. doi:10.1093/annonc/mdu042

10. Kawai M, Malone KE, Tang MT, et al. Height, body mass index (BMI), BMI change, and the risk of estrogen receptor-positive, HER2-positive, and triple-negative breast cancer among women ages 20 to 44 years. Cancer. 2014;120(10):1548-1556. doi:10.1002/ cncr. 28601

11. Nindrea RD, Aryandono T, Lazuardi L, et al. Association of overweight and obesity with breast cancer during premenopausal period in asia: a meta-analysis. Int J Prev Med. 2019;10(1):192. doi:10.4103/ijpvm.IJPVM_372_18

12. Patel V, James M, Frampton C, et al. Body mass index and outcomes in breast cancer treated with breast conservation. Int J Radiat Oncol Biol Phys. 2020;106(2):369-376. doi:10.1016/j.ijrobp.2019.09.049

13. Blair CK, Wiggins CL, Nibbe AM, et al. Obesity and survival among a cohort of breast cancer patients is partially mediated by tumor characteristics. NPJ Breast Cancer. 2019;5(1):33. doi:10.1038/ s41523-019-0128-4

14. Picon-Ruiz M, Morata-Tarifa C, Valle-Goffin JJ, et al. Obesity and adverse breast cancer risk and outcome: mechanistic insights and strategies for intervention. CA Cancer J Clin. 2017;67(5):378-397. doi: $10.3322 /$ caac. 21405
15. Yan M, Wang C, He B, et al. Aurora-A kinase: a potent oncogene and target for cancer therapy. Med Res Rev. 2016;36(6):1036-1079. doi: 10.1002/med.21399

16. Lykkesfeldt AE, Iversen BR, Jensen MB, et al. Aurora kinase A as a possible marker for endocrine resistance in early estrogen receptor positive breast cancer. Acta Oncol. 2018;57(1):67-73. doi:10.1080/ 0284186X.2017.1404126

17. Cheng L, Wang H, Guo K, et al. Reversine, a substituted purine, exerts an inhibitive effect on human renal carcinoma cells via induction of cell apoptosis and polyploidy. Onco Targets Ther. 2018;11:1025-1035. doi:10.2147/OTT.S158198

18. Sun B, Li J, Shao D, et al. Adipose tissue-secreted miR-27a promotes liver cancer by targeting FOXO1 in obese individuals. Onco Targets Ther. 2015;8:735-744. doi:10.2147/OTT.S80945

19. Miller LD, Smeds J, George J, et al. An expression signature for p53 status in human breast cancer predicts mutation status, transcriptional effects, and patient survival. Proc Natl Acad Sci U S A. 2005;102 (38):13550-13555. doi:10.1073/pnas.0506230102

20. Toro AL, Costantino NS, Shriver CD, et al. Effect of obesity on molecular characteristics of invasive breast tumors: gene expression analysis in a large cohort of female patients. BMC Obes. 2016;3 (1):22. doi:10.1186/s40608-016-0103-7

21. Ritter A, Kreis NN, Roth S, et al. Restoration of primary cilia in obese adipose-derived mesenchymal stem cells by inhibiting Aurora A or extracellular signal-regulated kinase. Stem Cell Res Ther. 2019;10(1):255. doi:10.1186/s13287-019-1373-z

22. Yang M, Qin X, Qin G, et al. The role of IRAK1 in breast cancer patients treated with neoadjuvant chemotherapy. Onco Targets Ther. 2019;12:2171-2180. doi:10.2147/OTT.S185662

23. Kamran M, Long ZJ, Xu D, et al. Aurora kinase A regulates survivin stability through targeting FBXL7 in gastric cancer drug resistance and prognosis. Oncogenesis. 2017;6(2):e298. doi:10.1038/oncsis.20 16.80

24. Kolb R, Kluz P, Tan ZW, et al. Obesity-associated inflammation promotes angiogenesis and breast cancer via angiopoietin-like 4 . Oncogene. 2019;38(13):2351-2363. doi:10.1038/s41388-018-05 92-6

25. Wellberg EA, Kabos P, Gillen AE, et al. FGFR1 underlies obesity-associated progression of estrogen receptor-positive breast cancer after estrogen deprivation. JCI Insight. 2018;3(14):120594. doi:10.1172/jci.insight.120594

26. Lee M, Lee E, Jin SH, et al. Leptin regulates the pro-inflammatory response in human epidermal keratinocytes. Arch Dermatol Res. 2018;310(4):351-362. doi:10.1007/s00403-018-1821-0

27. Kim C, Seedorf GJ, Abman SH, et al. Heterogeneous response of endothelial cells to insulin-like growth factor 1 treatment is explained by spatially clustered sub-populations. Biol Open. 2019;8(11): bio045906. doi:10.1242/bio.045906

28. Mesic A, Rogar M, Hudler P, et al. Characterization and risk association of polymorphisms in Aurora kinases A, B and C with genetic susceptibility to gastric cancer development. BMC Cancer. 2019;19 (1):919. doi:10.1186/s12885-019-6133-z

29. Mesic A, Markocic E, Rogar M, et al. Single nucleotide polymorphisms rs911160 in AURKA and rs2289590 in AURKB mitotic checkpoint genes contribute to gastric cancer susceptibility. Environ Mol Mutagen. 2017;58(9):701-711. doi:10.1002/em.22129

30. Liao Y, Liao Y, Li J, et al. Polymorphisms in AURKA and AURKB are associated with the survival of triple-negative breast cancer patients treated with taxane-based adjuvant chemotherapy. Cancer Manag Res. 2018;10:3801-3808. doi:10.2147/CMAR.S174 735

31. Li Y, Zhou W, Tang K, et al. Silencing Aurora A leads to re-sensitization of breast cancer cells to taxol through downregulation of SRC-mediated ERK and mTOR pathways. Oncol Rep. 2017;38 (4):2011-2022. doi:10.3892/or.2017.5908 
32. Mancini M, De Santis S, Monaldi C, et al. Hyper-activation of Aurora kinase a-polo-like kinase 1-FOXM1 axis promotes chronic myeloid leukemia resistance to tyrosine kinase inhibitors. J Exp Clin Cancer Res. 2019;38(1):216. doi:10.1186/s13046-019-1197-9

33. Marumoto T, Honda S, Hara T, et al. Aurora-A kinase maintains the fidelity of early and late mitotic events in HeLa cells. J Biol Chem. 2003;278(51):51786-51795. doi:10.1074/jbc.M306275200

34. Hole S, Pedersen AM, Lykkesfeldt AE, et al. Aurora kinase A and $\mathrm{B}$ as new treatment targets in aromatase inhibitor-resistant breast cancer cells. Breast Cancer Res Treat. 2015;149(3):715-726. doi:10.1007/s10549-015-3284-8

35. Choi EK, Lim JA, Kim JK, et al. Cyclin B11 stability is increased by interaction with BRCA1, and its overexpression suppresses the progression of BRCA1-associated mammary tumors. Exp Mol Med. 2018;50(10):136. doi:10.1038/s12276-018-0169-z

36. Sabbaghi M, Gil-Gómez G, Guardia C, et al. Defective Cyclin B11 Induction in trastuzumab-emtansine (T-DM1) acquired resistance in HER2-positive breast cancer. Clin Cancer Res. 2017;23(22):70 06-7019. doi:10.1158/1078-0432.CCR-17-0696
37. Sun X, Zhangyuan G, Shi L, et al. Prognostic and clinicopathological significance of Cyclin B1 expression in patients with breast cancer: a meta-analysis. Medicine (Baltimore). 2017;96(19):e6860. doi:10.10 97/MD.0000000000006860

38. Korobeynikov V, Borakove M, Feng Y, et al. Combined inhibition of Aurora A and p21-activated kinase 1 as a new treatment strategy in breast cancer. Breast Cancer Res Treat. 2019;177(2):369-382. doi:10.1007/s10549-019-05329-2

39. Gong X, Du J, Parsons SH, et al. Aurora A kinase inhibition is synthetic lethal with loss of the RB1 tumor suppressor gene. Cancer Discov. 2019;9(2):248-263. doi:10.1158/2159-8290.CD-18-0469

40. Tentler JJ, Ionkina AA, Tan AC, et al. p53 Family members regulate phenotypic response to Aurora Kinase A inhibition in triple-negative breast cancer. Mol Cancer Ther. 2015;14(5):1117-1129. doi:10.1158/ 1535-7163.MCT-14-0538-T

41. Hafner M, Mills CE, Subramanian K, et al. Multiomics profiling establishes the polypharmacology of FDA-approved CDK4/6 inhibitors and the potential for differential clinical activity. Cell Chem Biol. 2019;26(8):1067-1080. doi:10.1016/j.chembiol.2019.05.005

\section{Publish your work in this journal}

OncoTargets and Therapy is an international, peer-reviewed, open access journal focusing on the pathological basis of all cancers, potential targets for therapy and treatment protocols employed to improve the management of cancer patients. The journal also focuses on the impact of management programs and new therapeutic agents and protocols on patient perspectives such as quality of life, adherence and satisfaction. The manuscript management system is completely online and includes a very quick and fair peer-review system, which is all easy to use. Visit http://www.dovepress.com/ testimonials.php to read real quotes from published authors. 